\title{
Change of variables in the multiple Lebesgue integrals.
}

\author{
Masatsugu Tsujı. \\ (Received April 4, 1948)
}

Rademacher's theorem ${ }^{1)}$ on the change of variables in the multiple Lebesgue integrals, though very important, is not found in any book on the theory of functions of real variables, so that I will give a simple proof of it in the following lines.

Let $D$ be a domain in the $\left(x_{1}, \ldots \ldots, x_{n}\right)$-space and $\Delta$ be one in the $\left(u_{1}, \ldots \ldots, u_{n}\right)$ -space and $D$ be mapped on $\Delta$ topologically by

$$
\begin{array}{ll}
T: & u_{i}=f_{i}\left(x_{1}, \ldots, \ldots, x_{n}\right), \\
T^{-1}: & x_{i}=\varphi_{i}\left(u_{1}, \ldots ., u_{n}\right),(i=1,2, \ldots, \ldots, n),
\end{array}
$$

where $f_{i}$ and $\varphi_{i}$ are continuous in $D$ and $\Delta$ respectively.

If any measurable set in $D$ is mapped on a measurable set in $\Delta$, then $T$ is called a measurable mapping. It is well known' ${ }^{2)}$ that the necessary and sufficient condition that $T$ is a measurable mapping is that any null set ${ }^{3)}$ in $D$ is mapped on a null set in $\Delta$.

Theorem 1.4) If at every point $\left(x_{1}, \ldots \ldots, x_{n}\right) \in D$,

$$
\begin{gathered}
\varlimsup_{h_{1}^{2}+\ldots+h_{n}^{2} \rightarrow 0} \frac{\left|f_{i}\left(x_{1}+h_{1}, \ldots, x_{n}+h_{n}\right)-f_{i}\left(x_{1}, \ldots, x_{n}\right)\right|}{\sqrt{h_{1}^{2}+\ldots+h_{n}^{2}}} \\
\quad=L_{i}\left(x_{1}, \ldots, x_{n}\right)<\infty \quad(i=1,2, \ldots \ldots, n),
\end{gathered}
$$

then $T$ is a measurable mapping.

Proof. We define a set $A_{k}(\dot{k}=1,2, \ldots)$ of points $\left(x_{1}, \ldots, x_{n}\right)$ by the condition

(1) Rademacher: Über die partielle und totale Differentierbarkeit von Funktionen mehererer Veränderlichen und über die Transformation der Doppelintegrale. Math. Ann. 79.

(2) Rademacher: Eineindeutige Abbildung und Messbarkeit. Monathefte f. Math. u. Phys. 27 (1916). Carathéodory: Vorlesungen über reelle Funktionen. p. 354.

(3) A set is called a null set, if its Lebesgue measure is zero.

(4) Rademacher. 1. c. (1), (2). 
that for any $h_{1}, \ldots, h_{n}$, such that $h_{1}^{2}+\ldots+h_{n}^{2}<\frac{1}{k^{2}}$,

$$
\begin{gathered}
\left|f_{i}\left(x_{1}+h_{1}, \ldots, x_{n}+h_{n}\right)-f\left(x_{1}, \ldots, x_{n}\right)\right| \leqq k \sqrt{h_{1}{ }^{2}+\ldots+h_{n}{ }^{2}}, \\
(i=1,2, \ldots \ldots, n) .
\end{gathered}
$$

From the continuity of $f_{i}\left(x_{1}, \ldots, x_{n}\right)$, it follows that $A_{k}$ are closed sets and from (2)

$$
P=\sum_{k=1}^{\infty} A_{k} .
$$

Let $\left(x_{1}^{0}, \ldots, x_{n}^{0}\right) \in A_{k}$ and

$$
u_{i}=f_{i}\left(x_{1}^{0}+h_{1}, \ldots, x_{n}^{0}+h_{n}\right), \quad u_{n}^{0}=f_{i}\left(x_{1}^{0}, \ldots \ldots, x_{n}^{0}\right), \quad\left(h_{1}^{2}++h_{n}^{2}<\frac{1}{k^{2}}\right),
$$

then by (3),

$$
\sum_{i=1}^{n}\left(u_{i}-u_{i}^{0}\right)^{2} \leqq n k^{2}\left(h_{1}^{2}+\ldots+h_{n}^{2}\right) .
$$

Let $K(r)$ be the inside of a sphere of radius $r$ :

$$
K(r): \sum_{i=1}^{n}\left(x_{i}-x_{i}^{0}\right)^{2} \leqq r^{2}
$$

and $K^{\prime}(r)$ be its image in $\Delta$ by (1), then by (6),

$$
\sum_{i=1}^{n}\left(u_{i}-u_{i}^{0}\right)^{2} \leqq(\sqrt{n} k r)^{2},
$$

so that $K^{\prime}(r)$ is contained in a sphere of radius $\sqrt{n} k r$.

Since

$$
m K(r)=C r^{n},\left(C=\frac{\pi^{\frac{n}{2}}}{\Gamma\left(1+\frac{n}{2}\right)}\right),
$$

we have

$$
m K^{\prime}(r) \leqq M m K(r), \quad \text { if } \quad r \leqq \frac{1}{k},(M=\sqrt{n} k)^{n} .
$$

To prove that $T$ is a measurable mapping, it suffices to prove that any null set $e$ in $D$ is mapped on a null set $e^{\prime}$ in $\Delta$.

Let $e \subset D, \quad m e=0$ and put $e_{k}=e A_{k}$, then $m e_{k}=0$ and 


$$
e^{\prime}=\sum_{k=1}^{\infty} e_{k}, \quad e^{\prime}=\sum_{k=1}^{\infty} e_{k}^{\prime},
$$

where $e^{\prime}, e_{k}^{\prime}$ are images of $e, e_{k}$ in $\Delta$ respectively. We will prove that $m e_{k}^{\prime}=0$. Since $m e_{k}=0$, there exists for any $\varepsilon>0$ an open set $O$, such that $e_{k} \subset O, m O<\varepsilon$. We express $O$ as a sum of enumerably infinite number of non-overlapping cubes $\left\{\Delta_{i}\right\}$,

$$
O=\sum_{i=1}^{\infty} \Delta_{i}, \quad m O=\sum_{i=1}^{\infty} m \Delta_{i}<\varepsilon .
$$

Let $K_{i}$ be the inside of a sphere which is concentric with $\Delta_{i}$ and passes through the vertices of $\Delta_{i}$, then

$$
m K_{i} \leqq M^{\prime} m \Delta_{i}, \quad\left(M^{\prime}=\text { const. }\right)
$$

By taking $\Delta_{i}$ sufficiently small, we may assume that all $K_{i}$ are contained in $O$ and their radii are less than $\frac{1}{k}$. Let $K_{i}^{\prime}$ be the image of $K_{i}$ in $\Delta$. If $K_{i}$ has common points with $e_{k}$, then, since its radius is less than $\frac{1}{k}$, we have from $(9), m K^{\prime}{ }_{i} \leqq M m K_{i}$. Hence if we denote the sum of $K_{i}$, which have comnon points with $e_{k}$ by $\sum_{i}^{\prime} K_{i}$, then

$$
e_{k} \subset \sum_{i}^{\prime} K_{i}, \quad e_{i}^{\prime} \subset \sum_{i}^{\prime} K_{i}^{\prime},
$$

hence from (9), (12), (11),

$$
m{ }^{*}{ }^{e_{k}}{ }^{\prime} \leqq \sum_{i}{ }^{\prime} m K_{i}^{\prime} \leqq M \sum_{i} m K_{i} \leqq M M^{\prime} \quad \sum_{i}^{\prime} m \Delta_{i}<M M^{\prime} \varepsilon,
$$

where $m^{*} e_{k}{ }^{\prime}$ is the outer measure of $e_{k}^{\prime}$. Since $\varepsilon$ is arbitrary, we have $m e^{\prime}{ }_{k}=0$ and hence $m e^{\prime}=0$ from (10).

.Hence $T$ is a measurable mapping.

2.

$f\left(x_{1}, \ldots, \ldots, x_{n}\right)$ is said totally differentiable in Stolz's sense at $\left(x_{1}^{0}, \ldots \ldots, x_{n}{ }^{0}\right)$, if $f\left(x_{1}, \ldots, \ldots, x_{n}\right)$ is expressed in the neighbourhood of $\left(x_{1}{ }^{0}, \ldots \ldots, x_{n}{ }^{0}\right)$ in the form :

$$
\begin{gathered}
f\left(x_{1}{ }^{0}+h_{1}, \ldots \ldots, x_{n}{ }^{0}+h_{n}\right)=f\left(x^{0}, \ldots \ldots, x^{0}\right)+\sum_{k=1}^{n} \frac{\partial f\left(x_{1}{ }^{0}, \ldots, x_{n}^{0}\right)}{\partial x_{k}{ }^{0}} h_{k} \\
+D\left(h_{1}, \ldots \ldots, h_{n}\right),
\end{gathered}
$$

where 


$$
\lim _{h_{1}^{2}+\cdots+h^{2} n \rightarrow 0} \frac{\left|D\left(h_{1}, \ldots, h_{n}\right)\right|}{\sqrt{h_{1}^{2}++h_{n}^{2}}}=0 .
$$

If in (1), $f_{i}\left(x_{1}, \ldots, x_{n}\right) \quad(i=1,2, \ldots, n)$ satisfy the condition (2) at every point of $D$, then $f_{i}\left(x_{1}, \ldots, x_{n}\right)$ are totally differentiable almost-everywhere in $D^{5)}$. Hence $\frac{\partial f_{i}}{\partial x^{k}}$ and so

$$
J\left(x_{1}, \ldots, x_{n}\right)=\left|\begin{array}{l}
\frac{\partial f_{1}}{\partial x_{1}}, \ldots, \frac{\partial f_{1}}{\partial x_{n}} \\
\ldots \ldots, \ldots, \ldots, \ldots \\
\ldots \ldots, \ldots, \ldots, \ldots \\
\frac{\partial f_{n}}{\partial x_{1}}, \ldots, \frac{\partial f_{n}}{\partial x_{n}}
\end{array}\right|
$$

exists almost everywhere in $D$ and since $f_{i}$ are continuous, $\frac{\partial f_{i}}{\partial x_{k}}$ and hence $J\left(x_{1}, \ldots, x_{n}\right)$ is measurable in $D$.

Theorem 2. Let $f_{i}\left(x_{1}, \ldots, x_{n}\right)(i=1,2, \ldots, n)$ satisfy the condition (2) at every point of $D$ and $F\left(u_{1}, \ldots, u_{n}\right)$ be integrable in $\Delta$, then $F\left(f_{1}\left(x_{1}, \ldots, x_{n}\right), \ldots, f_{n}\left(x_{1}, \ldots, x_{n}\right)\right) \cup\left(x_{1}, \ldots x_{n}\right) \mid$ is measurable in $D$ and

$$
\begin{array}{r}
\int_{\Delta} \ldots \int F\left(u_{1}, \ldots, u_{n}\right) d u_{1} \ldots d u_{n}=\int_{D} \ldots \int F\left(f_{1}\left(x_{1}, \ldots, x_{n}\right), \ldots, f_{n}\left(x_{1}, \ldots, x_{n}\right)\right) \\
\left|J\left(x_{1}, \ldots, x_{n}\right)\right| d x_{1}, \ldots d x_{n} .
\end{array}
$$

Proof. Since $f_{i}\left(x_{1}, \ldots, x_{n}\right)$ are totally differentiable almost everywhere in $D$, we may assume that $(o, \ldots, o) \in D, f_{i}(o, \ldots, \ldots, o)=0$ and $f_{i}\left(x_{1}, \ldots, x_{n}\right)$ are totally differentiable at $(o, \ldots, o)$, so that in the neighbourhood of $(o, \ldots o),(1)$ can be expressed in the form:

$$
\left.\begin{array}{l}
u_{1}=f_{1}\left(x_{1}, \ldots, x_{n}\right)=a_{11} x_{1}+\ldots+a_{1 n} x_{n}+D_{1}\left(x_{1}, \ldots, x_{n}\right), \\
u_{n}=f_{n}\left(\ddot{x}_{1}, \ldots x_{n}\right)=a_{n 1} x_{1}+\ldots+a_{n n} x_{n}+D_{n}\left(x_{1}, \ldots, x_{n}\right)
\end{array}\right\},
$$

where $\quad a_{i k}=\left(\frac{\partial f_{i}\left(x_{1}, \ldots, x_{n}\right)}{\partial x_{k}}\right)_{x_{1}=\ldots=x_{n}=0}$

$$
\lim _{x_{1}{ }^{2}+\cdots+x^{2}{ }_{n} \rightarrow 0} \frac{\left|D_{i}\left(x_{1}, \ldots \ldots, x_{n}\right)\right|}{\sqrt{x_{1}^{2}+\ldots+x_{n}^{2}}}=0, \quad(i=1,2, \ldots, n),
$$

(5) Rademacher: 1.c. (1). Saks: Theory of the integral. p. 311.

(6) Rademcher: 1.c. (1). 
so that

$$
J(o, \ldots, o)=\left|\begin{array}{r}
a_{11}, \ldots \ldots, a_{1 n} \\
\ldots \ldots \ldots \ldots . . \\
a_{n 1}, \ldots \ldots, a_{n n}
\end{array}\right|
$$

Let $K(r)$ be the inside of a sphere of radius $r$ :

$$
K(r): x_{1}{ }^{2}+\ldots+x^{2}{ }_{n} \leqq r^{2}
$$

and $K^{\prime}(r)$ be its image in $\Delta$ by $(14)$. Then we will prove that

$$
\lim _{r \rightarrow 0} \frac{m K^{\prime}(r)}{m K(r)}=|\mathcal{}(o, \ldots, o)|
$$

To prove this, we associate to (14) an affine transformation:

$$
\left.\begin{array}{c}
v_{1}=a_{11} x_{1}+\ldots+a_{1 n} x_{n} \\
\ldots \ldots \ldots \ldots \ldots \\
v_{n}=a_{n 1} x_{1}+\ldots+a_{n n} x_{n} .
\end{array}\right\} .
$$

Then by (15),

$$
\sum_{i=1}^{n}\left(u_{i}-v_{i}\right)^{2} \leqq \varepsilon^{2}\left(x_{1}{ }^{2}+\ldots+x_{n}^{2}\right)
$$

where $\varepsilon \rightarrow 0$ with $x_{1}{ }^{2}+\ldots+x_{n}{ }^{2} \rightarrow 0$.

We have two cases to consider according as $J(o, \ldots o)=0$ or $J(o, \ldots, o) \neq 0$. (i) $\quad J(0, \ldots, 0)=0$.

In this case, $\left(x_{1}, \ldots, x_{n}\right)$-space is mapped on a linear sub-space of at most $(n-1)$-dimensions in $\left(v_{1}, \ldots, v_{n}\right)$-space by $(19)$, hence from $(20)$, we have easily $m K^{\prime}(r) \leqq \varepsilon r^{n}$, where $\varepsilon \rightarrow 0$ with $r \rightarrow o$, hence from (8), we have $m K^{\prime}(r) \leqq \delta m K(r)$, where $\delta \rightarrow 0$ with $r \rightarrow 0$, so that

$$
\lim _{r \rightarrow 0} \frac{m K(r)}{m K(r)}=o=|J(o, \ldots, o)|
$$

(ii) $\quad J(0, \ldots, o) \neq 0$.

In this case, we can solve (19) with respect to $x_{i}$, such that

$$
\left.\begin{array}{c}
x_{1}=b_{11} v_{1}+\ldots . .+b_{1 n} v_{n}, \\
\ldots \ldots \ldots \ldots \ldots \ldots . . . \\
x_{n}=b_{n 1} v_{1}+\ldots . .+b_{n n} v_{n}
\end{array}\right\},
$$

where 


$$
\left|\begin{array}{c}
b_{11}, \ldots, b_{1 n} \\
\ldots \ldots, \ldots, \\
b_{n_{1}} \ldots, b_{n n}
\end{array}\right|=\left|\begin{array}{l}
a_{11}, \ldots, a_{1 n} \\
a_{n 1}, \ldots, a_{n n}
\end{array}\right|=\frac{1}{J(o, \ldots \ldots, o)} .
$$

Let $K(r)$ be mapped on $\Delta(r)$ by (19), then by (22), $\Delta(r):\left(b_{11} v_{1}+\ldots+b_{1 n} v_{n}\right)^{2}+\ldots+\left(b_{n 1} v_{1}++\ldots+b_{n n} v_{n}\right)^{2}=\sum_{i, k}^{1, \ldots n} B_{i k} v_{i} v_{k} \leqq r^{2}$, where

$$
B=\left|\begin{array}{l}
B_{11}, \ldots \ldots, B_{1 n} \\
\ldots \ldots \ldots, \ldots, \\
B_{n_{1}}, \ldots \ldots, B_{n n}
\end{array}\right|=\left|\begin{array}{l}
b_{11}, \ldots \ldots, b_{1 n} \\
\ldots \ldots \ldots, \ldots \\
b_{n 1}, \ldots \ldots, b_{n n}
\end{array}\right|=\frac{1}{J^{2}(o \ldots \ldots, o)} .
$$

By a suitable orthogonal transformation, $\Delta(r)$ can be brought into the form :

$$
\Delta(r): \frac{\xi_{1}^{2}}{a_{1}^{2}}+\ldots+\frac{\xi_{n}^{2}}{a_{n}^{2}} \leqq r^{2}, \quad\left(a_{i}>0\right),
$$

where $\lambda_{1}=\frac{1}{a_{1}^{2},}, \ldots, \lambda_{n}=\frac{1}{a_{n}{ }^{2}}$ are the roots of the characteristic equation :

$$
B(\lambda)=\left|\begin{array}{c}
B_{11}-\lambda, \ldots \ldots, B_{1 n} \\
\ldots \ldots \ldots \ldots \ldots \ldots . . . \\
B_{n 1}, \ldots \ldots . B_{n n}-\lambda
\end{array}\right|=0,
$$

so that by $(25)$,

$$
\begin{aligned}
\frac{1}{\left(a_{1} \ldots a_{n}\right)^{2}} & =\lambda_{1} \ldots \lambda_{n}=B(o)=\frac{1}{J^{2}(o \ldots, o),}, \text { or } \\
a_{1} \ldots a_{n} & =|J(o, \ldots, o)| .
\end{aligned}
$$

Since by a transformation : $\xi_{i}=a_{i} X_{i}$, (26) can be transformed into the form : $X_{1}^{2}+\ldots+X_{n}{ }_{n} \leqq r^{2}$, we have from $(8)$,

$$
\dot{m} \Delta(r)=a_{1} \ldots a_{n} m K(r)=C \cdot a_{1} \ldots a_{n} r^{n} \text {. }
$$

If $\left(x_{1}, \ldots, x_{n}\right)$ lies on a sphere $: x^{2}+\ldots+x_{n}{ }^{2}=r^{2}$, then $\left(v_{1}, \ldots, v_{n}\right)$ lies on an ellipsoid : $\frac{\xi_{1}{ }^{2}}{a_{1}{ }^{2}}+\ldots+\frac{\xi_{n}{ }_{n}}{a^{2}{ }_{n}}=r^{2}$, so that by $(20),\left(u_{1}, \ldots, u_{n}\right)$ lies between two ellipsoids :

$$
\frac{\xi_{1}^{2}}{a_{1}^{2}}+\ldots+\frac{\xi_{n}^{2}}{a_{n}^{2}}=r^{2}(1-\delta)^{2}, \quad \frac{\xi_{1}{ }^{2}}{a_{1}{ }^{2}}+\ldots+\frac{\xi_{n}^{2}}{a_{n}^{2}}=r^{2}(1+\delta)^{2},
$$

where $\delta \rightarrow 0$ with $r \rightarrow o$. Hence we have from (27), (28), $C a_{1} \ldots a_{n} r^{n}(1-\delta)^{n} \leqq m K^{\prime}(r) \leqq C a_{1} \ldots a_{n} r^{n}(1+\delta)^{n}$, or $|J(o, \ldots, o)|(1-\delta)^{n} m K(r) \leqq m K^{\prime}(r) \leqq|J(o, \ldots, o)|(1+\delta)^{n} m K(r)$, so that 


$$
\lim _{r \rightarrow 0} \frac{m K^{\prime}(r)}{m K(r)}=|J(o, \ldots, o)|
$$

From (21), (29), we have (18).

By Theorem 1, the mapping $u_{i}=f_{i}\left(x_{1}, \ldots x_{n}\right)$ is a measurable mapping, so that any measurable set $e \subset D$ is mapped on a measurable set $e^{\prime} \subset \Delta$ and a null set in $D$ is mapped on a null set in $\Delta$. Hence if we put

$$
m \epsilon^{\prime}=\Phi(e) \text {, }
$$

then $\Phi(e)$ is an absolutely continuous additive set function defined on measurable sets in $D$, so that by Lebesgue's theorem,

$$
m e^{\prime}=\Phi(e)=\int \ldots \int_{e} D \Phi\left(x_{1}, \ldots, x_{n}\right) d x_{1}, \ldots d x_{n}
$$

where $D \Phi\left(x_{1}, \ldots, x_{n}\right)$ is the derivative of $\Phi(c)$. at $\left(x_{1}, \ldots x_{n}\right)$, which exists almost everywhere in $D$. From (18), we have $D \Phi\left(x_{1}, \ldots x_{n}\right)=\left|J\left(x_{1}, \ldots x_{n}\right)\right|$ almost everywhere in $D$, so that

$$
m e^{\prime}=\int \ldots \int_{e}\left|J\left(x_{1}, \ldots, x_{n}\right)\right| d x_{1} \ldots . . d x_{n} .
$$

Let $\epsilon_{0}$ be the set of $\left(x_{1} \ldots, x_{n}\right)$, such that $J\left(x_{1}, \ldots, x_{n}\right)=0$, then $e_{0}$ is measurable, so that from $(30)$,

$$
m e^{\prime}=\ddot{o}
$$

where $e_{0}^{\prime}$ is the image of $e_{0}$ in $\Delta$.

Now $\Delta-e_{0}^{\prime}$ is mapped on $D-e_{0}$ by $x_{i}=\varphi_{i}\left(u_{1}, \ldots, u_{n}\right)$. We will prove that this is a measurable mapping. To prove this, it suffices to prove that any null set $e^{\prime} \subset \Delta-e_{0}^{\prime}$ is mapped on a null set $e \subset D-e_{0}$.

Let $e^{\prime} \subset \Delta-e^{\prime}{ }_{0}$ be a null set and $e$ be its image in $D-e_{0}$. Since $m e^{\prime}=o$, there exists a $G_{\delta}$-set $H^{\prime}$ in $\Delta$, such that

$$
e^{\prime} \subset H^{\prime}, \quad m H^{\prime}=0 .
$$

Let $H$ be the image of $H^{\prime}$ in $D$, then $H$ is a $G_{\delta}$-set, so that by (30),

$$
o=m H^{\prime}=\int_{H} \ldots \int U\left(x_{1}, \ldots, x_{n}\right) \mid d x_{1} \ldots \ldots d x_{n} .
$$

Hence $J\left(x_{1}, \ldots, x_{n}\right)=0$ almost everywhere in $H$. From this we conclude that $m\left(H-H e_{0}\right)=o$. Since $e \in H-H e_{0}$, we have $m e=o$, q.e.d. 
Hence any measurable set in $\Delta-e_{0}^{\prime}$ is mapped on a measurable set in $D-e_{0}$.

Let $F\left(u_{1}, \ldots, u_{n}\right)$ be a measurable function in $\Delta$ and for any real number $\alpha$, let $E_{\alpha}^{\prime}$ be the set of $\left(u_{1}, \ldots, u_{n}\right) \in \Delta-e^{\prime}{ }_{0}$, such that

$$
F\left(u_{1}, \ldots, u_{n}\right)>\alpha \text {. }
$$

Then $E_{\alpha}^{\prime}$ is measurable, so that its image $E_{\alpha} \subset D-e_{0}$ by $x_{i}=\varphi_{i}\left(u_{1}, \ldots, u_{n}\right)$ is measurable. Evidently $E_{\alpha}$ is the set of $\left(x_{1}, \ldots, x_{n}\right) \in D-e_{0}$, such that

$$
F\left(f_{1}\left(x_{1}, \ldots, x_{n}\right), \ldots, f_{n}\left(x, \ldots, x_{n}\right)\right)>\alpha .
$$

Hence $F\left(f_{1}\left(x_{1}, \ldots, x_{n}\right), \ldots, f_{n}\left(x_{1}, \ldots, x_{n}\right)\right)$ and so

$$
F\left(f_{1}\left(x_{1}, \ldots, x_{n}\right), \ldots f_{n}\left(x_{1}, \ldots, x_{n}\right)\right)\left|J\left(x_{1}, \ldots, x_{n}\right)\right|
$$

is measurable in $D-e_{0}$. Since $J\left(x_{1}, \ldots, x_{n}\right)=0$ in $e_{0},(34)$ is measurable in D.

First we suppose that $F\left(u_{1}, \ldots, u_{n}\right)$ is bounded and $A<F\left(u_{1}, \ldots u_{n}\right)<B$ in $\Delta$. Let

$$
\begin{gathered}
A=l_{0}<l_{1}<\ldots<l_{p+1}=B \quad\left(l_{i+1}-l_{i}<\varepsilon\right), \\
E_{i}^{\prime}=E\left(l_{i} \leqq F\left(u_{1}, \ldots, u_{n}\right)<l_{i+1}\right), \text { where } E=\Delta-e_{0}^{\prime},
\end{gathered}
$$

and $E_{i}$ be the image of $E_{i}^{\prime}$ in $D-e_{0}$, then $E_{i}^{\prime}, E_{i}$ are measurable and

$$
\sum_{i=0}^{p} E_{i}^{\prime}=\Delta-e_{0}^{\prime}, \quad \sum_{i=0}^{p} E_{i}=D-e_{0} .
$$

By $(30)$,

$$
m E_{i}^{\prime}=\int_{E i} \ldots \int U\left(x_{1}, \ldots, x_{n}\right) \mid d x_{1} \ldots d x_{n}
$$

We put

$$
\begin{gathered}
S=\sum_{i=0}^{p} l_{i} m E_{i}^{\prime}=\sum_{i=0}^{p} l_{i} \int_{F i} \ldots \int\left|J\left(x_{1}, \ldots, x_{n}\right)\right| d x_{1} \ldots d x_{n} \\
I=\int_{D} \ldots \int F\left(f_{1}, \ldots, f_{n}\right)\left|J\left(x_{1}, \ldots, x_{n}\right)\right| d x_{1} \ldots d x_{n}=\int_{D-e_{0}} \ldots \int F\left(f_{1}, \ldots, f_{n}\right) \\
=\sum_{i=0}^{p} \int \ldots \int_{L_{i}} F\left(f_{1}, \ldots, f_{n}\right)\left|J\left(x_{1}, \ldots, x_{n}\right)\right| d x_{1} \ldots d x_{n}
\end{gathered}
$$

then 


$$
\begin{aligned}
& |I-S| \leqq \sum_{i=0}^{p} \int_{E i} \ldots \int\left|F\left(f_{1}, \ldots, f_{n}\right)-l_{i}\right|\left|J\left(x_{1}, \ldots, x_{n}\right)\right| d x_{1} \ldots d x_{n} \leqq \\
& \sum_{i=0}^{p} \int_{E i} \ldots \int\left(l_{i+1}-l_{i}\right)\left|J\left(x_{1}, \ldots, x_{n}\right)\right| d x_{1} \ldots . d x_{n} \leqq \varepsilon \int_{j, e^{\circ}} \ldots \int\left|J\left(x_{1}, \ldots x_{n}\right)\right| d x_{1}, \ldots d x_{n} \\
& =\varepsilon \int_{j} \ldots \int\left|J\left(x_{1}, \ldots x_{n}\right)\right| d x_{1} \ldots d x_{n}=\varepsilon m \Delta .
\end{aligned}
$$

Since for $\varepsilon \rightarrow 0$.

$$
S \rightarrow \int_{\Delta-e^{\prime} 0} \ldots \int_{0} F\left(u_{1}, \ldots u_{n}\right) d u_{1} \ldots d u_{n}=\int_{\Delta} \ldots \int F\left(u_{1}, \ldots u_{n}\right) d u_{1}, \ldots d u_{n},
$$

we have from (39),

$$
\int \ldots \int_{\Delta} F\left(u_{1}, \ldots, u_{n}\right) d u_{1}, \ldots d u_{n}=\int \ldots \int F\left(f_{1}, \ldots, f_{n}\right)\left|J\left(x_{1}, \ldots, x_{n}\right)\right| d x_{1}, \ldots d x_{n} .(40)
$$

If $F\left(u_{1}, \ldots, u_{n}\right) \geqq 0$ in $\Delta$, then put

$$
F_{N}\left(u_{1}, \ldots, u_{n}\right)=\left[F\left(u_{1}, \ldots, u_{n}\right)\right]_{0}{ }^{v},
$$

where $F_{N}=F ;$ if $F \leqq N$ and $F=N$, if $F \geqq N$. Then from (40)

$$
\int_{\Delta} \ldots \int_{N} F_{N}\left(u_{1}, \ldots, u_{n}\right) d u_{1} \ldots d u_{n}=\int_{\ddot{D}} \ldots \int F_{N}\left(f_{1}, \ldots . ., f_{n}\right)\left|J\left(x_{1}, \ldots, x_{n}\right)\right| d x_{1} \ldots d x_{n} .
$$

If we make $N \rightarrow \infty$, then we have (40). In the general case we put $F=F_{1}-F_{2}$, where $F_{1}=\frac{|F|+F}{2} \geqq 0, \quad F_{2}=\frac{|F|-F}{2} \geqq o$ and apply (40) on $F_{1}$ and $F_{2}$ and we have (40).

Hence the theorem is completely proved.

Mathematical Institute,

Tokyo University. 